\title{
Elevated MMP9 expression in breast cancer is a predictor of shorter patient survival
}

\author{
Chitra Joseph ${ }^{1}$ (D) Mansour Alsaleem ${ }^{1,2} \cdot$ Nnamdi Orah $^{1} \cdot$ Pavan L. Narasimha $^{1} \cdot$ Islam M. Miligy ${ }^{1} \cdot$ Sasagu Kurozumi $^{1}$. \\ Ian O. Ellis ${ }^{1}$ - Nigel P. Mongan ${ }^{4,5} \cdot$ Andrew R. Green ${ }^{1} \cdot$ Emad A. Rakha ${ }^{1,3,6}$
}

Received: 26 November 2019 / Accepted: 5 May 2020 / Published online: 22 May 2020

(c) The Author(s) 2020

\begin{abstract}
Purpose MMP9 is a matricellular protein associated with extracellular matrix (ECM) remodelling, that promotes tumour progression, and modulates the activity of cell adhesion molecules and cytokines. This study aims to assess the prognostic value of MMP9 and its association with cytoskeletal modulators in early-stage invasive breast cancer (BC).

Methods MMP9 expression was evaluated by immunohistochemistry using a well-characterised series of primary BC patients with long-term clinical follow-up. Association with clinicopathological factors, patient outcome and ECM remodelling BC-biomarkers were investigated. METABRIC dataset, BC-GenExMiner v4.0 and TCGA were used for the external validation of $M M P 9$ expression. GSEA gene enrichment analyses were used to evaluate $M M P 9$ associated pathways.

Results MMP9 immunopositivity was observed in the stroma and cytoplasm of BC cells. Elevated MMP9 protein levels were associated with high tumour grade, high Nottingham Prognostic Index, and hormonal receptor negativity. Elevated MMP9 protein expression correlated significantly with cytokeratin 17 (Ck17), Epidermal Growth Factor Receptor (EGFR), proliferation (Ki67) biomarkers, cell surface adhesion receptor (CD44) and cell division control protein 42 (CDC42). Cytoplasmic MMP9 expression was an independent prognostic factor associated with shorter BC-specific survival. In the external validation cohorts, $M M P 9$ expression was also associated with poor patients' outcome. Transcriptomic analysis confirmed a positive association between $M M P 9$ and ECM remodelling biomarkers. GSEA analysis supports MMP9 association with ECM and cytoskeletal pathways.

Conclusion This study provides evidence for the prognostic value of MMP9 in BC. Further functional studies to decipher the role of MMP9 and its association with cytoskeletal modulators in BC progression are warranted.
\end{abstract}

Keywords MMP9 $\cdot$ Breast cancer $\cdot$ ECM remodelling $\cdot$ Prognosis

\section{Abbreviations}

BC Breast cancer

BCSS BC-specific survival

CI Confidence intervals

Electronic supplementary material The online version of this article (https://doi.org/10.1007/s10549-020-05670-x) contains supplementary material, which is available to authorized users.

Emad A. Rakha

Emad.Rakha@nottingham.ac.uk

1 Nottingham Breast Cancer Research Centre, School of Medicine, University of Nottingham, Nottingham, UK

2 Faculty of Applied Medical Sciences, Onizah Community College, Qassim University, Qassim, Saudi Arabia

3 Histopathology Department, Faculty of Medicine, Menoufia University, Shibin El Kom, Egypt
DGE Differential gene expression

ER Oestrogen

HR Hazard ratio

HER2 Human epidermal growth factor receptor 2

METABRIC Molecular taxonomy of breast cancer international consortium

NPI Nottingham Prognostic Index

PR Progesterone

4 Department of Pharmacology, Weill Cornell Medicine, New York 10065, USA

5 Faculty of Medicine and Health Sciences, University of Nottingham, Nottingham LE12 5RD, UK

6 Division of Cancer and Stem Cells, Department of Histopathology, School of Medicine, The University of Nottingham and Nottingham University Hospitals NHS Trust, Nottingham City Hospital, Nottingham NG5 1PB, UK 
TCGA The cancer genome atlas

TNBC Triple negative breast cancer

\section{Introduction}

Matrix metalloproteinases (MMPs) are a family of proteases that have multiple biological functions in cancer development and progression and are abundantly up regulated in breast cancer (BC). MMP9, also known as gelatinase $\mathrm{B}$, plays an important role in extracellular matrix (ECM) remodelling, protein cleavage, and is associated with tumour invasion, metastasis and modulation of tumour microenvironment $[1,2]$. MMP9 has the capability to degrade collagens, including Type IV collagen [3], which plays a role in basement membrane degradation promoting migration, invasion and metastases.

MMP9 is secreted as an inactive pro-enzyme and activation of latent MMP9 is the critical step in its regulation [4]. In vitro and in vivo experiments in human and experimental models of cancers reveal that the increased MMP9 expression is related to tumour progression [5, 6]. MMP9 expression is regulated by several molecular pathways such as extracellular signal-regulated kinase (ERK), mitogenactivated protein kinase (MAPK), and phosphoinositide3-kinase-protein kinase (PI3K); pathways recognised to be altered in $\mathrm{BC}$ [7]. In $\mathrm{BC}$, increased epidermal growth factor receptor (EGFR) expression, which is a poor prognostic marker, is implicated with up-regulation of MMP9 [8]. The regulation of the MMPs, particularly MMP9, by p53 has also been documented [9].

MMP9 is activated by the Cell Division Cycle 42 (CDC42), a Rho GTPase family member, which plays a major role in ECM adhesion and cytoskeletal organisation. Elevated CDC42 expression in BC dysregulates the epithelial architecture, which may initiate oncogenes. CDC42 gene silencing studies in $\mathrm{BC}$ xenografts showed that CDC42 knockdown decreased the tumour cell invasion and metastasis in vivo [10]. In addition, CDC42 activation stimulated trafficking of membrane-type 1 matrix metalloproteinase (MT1-MMP) in BC cells [11].

CD44 is a transmembrane glycoprotein cell surface adhesion receptor that promotes the secretion of active MMP9. $M M P 9$ gene silencing is shown to change the expression of CD44 and significantly decreases migration and invasion of tumour cells [12]. Increased $M M P 9$ mRNA expression was also observed in $\mathrm{CD} 44^{+} \mathrm{BC}$ cells compared to $\mathrm{CD} 44^{-}$cells. In vitro experiments showed that, inhibition of the CD44MMP axis may provide therapeutic targets for reducing the tumour spread which further establishes a positive association between MMP9 and CD44 expression [10]. Thus, these studies support a role for CD44 in regulating MMP9 and is strongly associated with aggressively behaving tumours. In addition, MMP9 is part of the Rosetta poor-prognosis signature for BC [13] and in silico analysis of BC DNA microarray datasets also showed a positive association of MMP9 with poor outcomes [14]. For these reasons in this study we investigated the association between MMP9, cytoskeletal modulators, and clinicopathological factors of $\mathrm{BC}$ at the protein and mRNA levels using multiple well-characterised early-stage $\mathrm{BC}$ cohorts.

\section{Materials and methods}

\section{Study cohort characteristics}

This study obtained ethics approval by the North West-Greater Manchester Central Research Ethics Committee under the title; Nottingham Health Science Biobank (NHSB), reference number 15/NW/0685. All samples from Nottingham used in this study were pseudo-anonymised and collected prior to 2006 and stored in compliance with the UK Human Tissue Act. MMP9 protein expression was evaluated using a well-characterised cohort of early-stage (operable) primary invasive BC $(n=675)$ treated in Nottingham University Hospital NHS Trust as previously described [15]. Clinical and pathological data of patients (including hormone receptor status, histological tumour type, tumour grade, tumour size, lymph node status, Nottingham Prognostic Index and lymphovascular invasion (LVI)) were available. Tumour types were revisited and coded according to the recent WHO blue book [16]. BC-specific survival (BCSS) was maintained on a prospective basis. The expression of a large panel of $\mathrm{BC}$ progression/metastasis-related biomarkers, including the Ki67 [17], EGFR [18], CDC42 [19], CD44 [20], PIK3CA [21] and basal marker (cytokeratin 5/6 and 17) [22], was also studied. BC molecular subtypes based on the IHC profile of Oestrogen Receptor (ER), Progesterone Receptor (PR) and Human Epidermal Growth Factor 2 (HER2) were defined as previously described [23]: Luminal A: ER+/HER2- Low Proliferation (Ki67 < 10\%), Luminal B: ER+/HER2- High Proliferation (Ki67 $\geq 10 \%)$ or ER +/ HER2 +, HER2-positive class: HER2 + regardless of ER status, Triple Negative Breast Cancer (TNBC): exhibiting negative expression of ER, PR, and HER2.

The clinicopathological significance of $M M P 9$ mRNA expression, copy number alterations, differential gene expression analysis (DGE), and pathway analysis were assessed using the Molecular Taxonomy of Breast Cancer International Consortium (METABRIC) dataset $(n=1980$ BC cases) [24]. External validation was performed using the Breast Cancer Gene-Expression Miner v4.0 (BC-GenExMiner v4.0) $[15,25]$ and The Cancer Genome Atlas (TCGA) [26] as previously described. 


\section{Immunohistochemistry}

Specificity of MMP9 antibody was validated by western blotting prior to immunohistochemistry. Cell lysates blots of HEK293 and MCF7 cell lines which were used as positive and negative controls respectively (obtained from the American Type Culture Collection; Rockville, MD, USA) were incubated with anti-MMP9 antibody (Rabbit monoclonal [EP1254], Abcam) at 1:800 dilution for overnight $\left(4{ }^{\circ} \mathrm{C}\right)$ and bands were detected using fluorescent secondary antibodies at $(1: 15,000)$ (IR Dye $800 \mathrm{CW}$ donkey antirabbit and 680RD donkey anti-mouse, LI-COR Biosciences, UK). Mouse $\beta$-Actin (A5441, Sigma-Aldrich; Clone AC-15; Sigma, UK) at 1:5000 was used as a house-keeping protein. Blocking and visualisation were done as previously documented [27]. The specificity of the antibody was validated with a single specific band at the predicted molecular weight (70 kDa, Supplementary Fig. 1a).

Tumour samples were arrayed onto tissue microarrays (TMAs) as previously described [28]. Full-face BC tissue sections and TMAs were immunoassayed using Novolink Max Polymer Detection system (Leica, Newcastle, UK). In brief, $4 \mu \mathrm{m}$ BC tissue sections were deparaffinised with xylene and rehydrated through $100 \%$ ethanol. Heat-induced (pH6) citrate antigen retrieval was performed and MMP9 antibody (1:100) was incubated for overnight at $4{ }^{\circ} \mathrm{C} .3-3^{\prime}$ Diaminobenzidine tetrahydrochloride (Novolink DAB substrate buffer plus) was used as the chromogen. Slides were counterstained with Novolink haematoxylin for 6 min, dehydrated and cover slipped. Normal kidney tissue was used as a positive tissue control, whereas no primary antibody was used as a negative control.

TMA slides were digitally scanned at 20X magnification and viewed using NanoZoomer NDP viewer (Hamamatsu Photonics, Welwyn Garden City, UK). Both the percentage of staining and staining intensity of MMP9 cytoplasmic expression in invasive tumour cells and stromal cells were individually assessed to calculate the final histochemical score (H-score). Staining was double scored blindly by two researchers (NO and IMM) for $25 \%$ cores to assess interobserver concordance. Inter-observer agreement was determined, and the interclass correlation coefficient was 0.86 , indicating an excellent concordance between scorers. Discordant cases were re-scored by both observers and a final score was agreed.

\section{Statistical analysis}

IBM SPSS 24.0 (SPSS IBM Corp, Chicago, IL, USA) software was used for statistical analysis and reported in line with REMARK guidelines [29]. The MMP9 H-score was dichotomised into high and negative/low expression using the median cut-point value. Chi-squared test was used to evaluate the association between MMP9 expression and the clinicopathological parameters.

Kaplan-Meier analysis with log-rank test for significance was performed to assess BCSS. Cox multivariate analysis was performed to test independence from standard prognostic factors in BC (nodal stage, tumour grade, tumour size, ER level of expression, and Ki67). A $p$ value of $<0.05$ was considered significant.

Gene expression was analysed in the subset of METABRIC patients for which MMP9 expression was available. DGE between A) low Vs high MMP9 cytoplasmic expression, and B) low Vs high MMP9 stromal expression were calculated using the Robina implementation of Edge- $R$ statistical tool [30] and DGE with $>$ twofold-change, and adjusted $p$ values $<0.05$ were considered significant. The DGE were examined using the online WebGestalt platform and adjusted $p<0.01$ considered statistically significant [31, 32] and significantly enriched gene ontologies common for both cytoplasmic and stromal MMP9 protein expression. Furthermore, Venny 2.0 [33] was used to identify the overlapping DGEs common to both the cytoplasmic and stromal MMP9 protein expression.

\section{Results}

\section{MMP9 protein expression in BC}

Full-face BC tissue $(n=10)$ sections were used to evaluate the pattern of MMP9 protein expression prior to staining of TMAs. This showed uniformly weak MMP9 expression in normal glandular epithelium (Fig. 1a) and ductal carcinoma in situ (DCIS; Fig. 1b). There was slightly increased immunoreactivity of MMP9 observed in the co-existing invasive BC cells (Fig. 1c), in the intravascular tumour cell emboli (Fig. 1d), stromal expression (Fig. 1e) and Fig. 1f showing No Primary Antibody Control. MMP9 cytoplasmic and stromal protein expression $(\mathrm{C}+/ \mathrm{S}+)$ showed a positive correlation with $M M P 9$ mRNA (Spearman's coefficient 0.218; $p=0.027$ ), this association was confirmed using TCGA data [34, 35] (Supplementary Fig. 1b).

On BC TMAs, a variable degree of MMP9 protein expression was observed (Fig. 1g-k). The H-scores of both cytoplasmic and stromal expressions did not follow a normal distribution. Therefore, for dichotomisation into negative/low and high expression, the median $\mathrm{H}$-scores $(0$ and 50 , respectively) were used. Out of 675 informative TMA cores, $71 \%$ showed negative/low expression in the cytoplasm (Fig. 1g) while 29\% showed high expression (Fig. 1h). In the stroma 53\% showed negative/low expression (Fig. 1i), while $47 \%$ showed high expression (Fig. 1j). Positive immunoreactivity was observed in human kidney sections (Fig. 1k). 

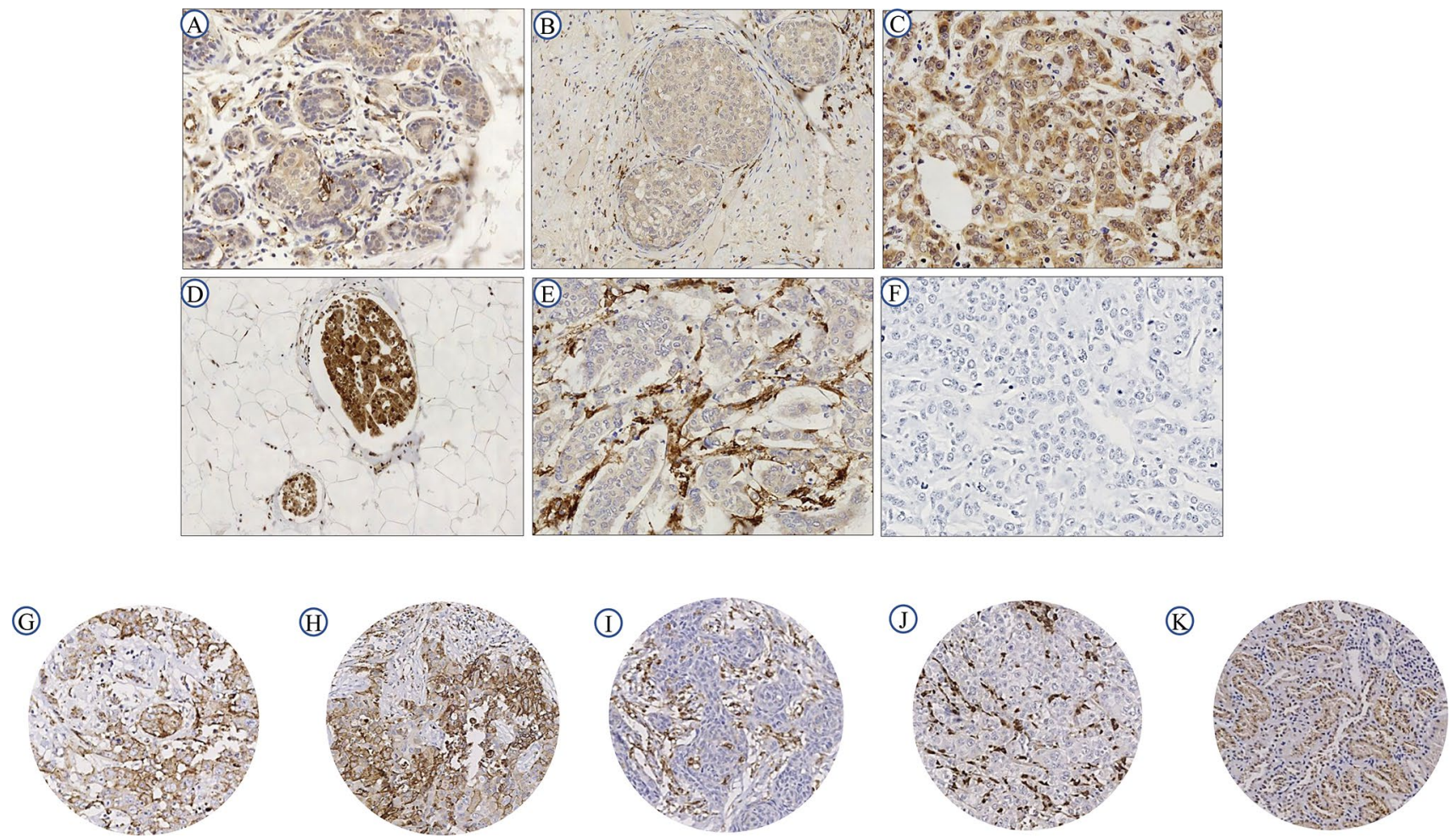

Fig. 1 Immunohistochemical expression of MMP9 in BC. Morphological characteristics of MMP9 immunohistochemistry in Full-face breast cancer tissue (a-f). a Normal mammary gland and b DCIS showing absent or weak MMP9 staining. Showing high immunoreactivity in $\mathbf{c}$ invasive, and $\mathbf{d}$ LVI positive tumour samples. Showing high MMP9 stromal staining (e) and (f) no primary antibody control

$\mathrm{H}$-scores for cytoplasmic and stromal staining showed a positive correlation (Spearman's coefficient $0.1 ; p=0.020$ ).

Elevated MMP9 cytoplasmic and stromal staining were associated with high tumour grade $(p<0.0001)$, poor Nottingham Prognostic Index (NPI) $(p<0.0001$; Table 1$)$, hormone receptor negativity $(p<0.01)$, among IHC subtypes associated with TNBC and HER2 + tumours $(p=0.002)$. High stromal MMP9 expression additionally showed association with LVI positivity ( $p=0.046$; Table 1). High MMP9 expression positively associated with proliferation marker Ki67 ( $p=0.004)$, signalling pathway associated markers; EGFR $(p=0.024)$ and PIK3CA $(p=0.039)$, cytoskeleton remodelling markers; CDC42 and CD44 $(p<0.003$ and $p=0.025$, respectively). High cytoplasmic MMP9 showed positive association with basal cytokeratin CK17 ( $p=0.001$; Table 2) and revealed a low expression in lobular and special type tumours $(p=0.003)$.

BCSS of patients with tumours expressing high cytoplasmic MMP9 was significantly shorter than that of the negative/low expression subgroup $(p=0.013 ; \mathrm{HR}=1.5 ; 95 \% \mathrm{CI}$ 1.1-2.0; Fig. 2a). Stromal MMP9 also showed a similar trend but did not reach significance $(p=0.058 ; \mathrm{HR}=1.3$; 95\%CI 0.9-1.8; Fig. 2b). Combined MMP9 cytoplasmic and (negative control) in invasive breast carcinoma. All images are at $50 \mu \mathrm{m}$. MMP9 protein expression in breast cancer TMA cores (g) and (h) showing low and high cytoplasmic $(\mathrm{C}+)$ expression while, $\mathbf{i}$ and $\mathbf{j}$ showing low and high stromal ( $\mathrm{S}+$ ) expression, respectively. The normal kidney tissue (k) section used as positive control. TMA cores are $100 \mu \mathrm{m}$ magnification

stromal (CS) survival analysis demonstrated that tumours with high cytoplasmic and stromal expression were associated with poor prognosis $(p=0.008 ; \mathrm{HR}=1.2 ; 95 \% \mathrm{CI}$ 1.0-1.4; Fig. 2c). In multivariate Cox regression analysis, cytoplasmic MMP9 expression on its own was an independent predictor of $\mathrm{BCSS}(p=0.026 ; \mathrm{HR}=1.6 ; 95 \%$ CI 1.1-2.3; Table 3). There was no association between MMP9 protein and outcome in Luminal A and B, TNBC or HER2 + subgroups.

\section{MMP9 genomic profiling}

Consistent with the results obtained for MMP9 protein expression, in the METABRIC and TCGA datasets, MMP9 copy number gain (14.0\%) and high mRNA expression (50.1\%) was associated with negative ER and PR status, high histological grade, and poor NPI (all; $p<0.01$; Table 4). $M M P 9$ copy number gain and high $M M P 9$ mRNA expression was associated with poor prognostic METABRIC Integrative clusters [24] such as 1,5 and $9(p<0.0001)$. Associations between $M M P 9$ copy number alterations, $m R N A$ expression and clinicopathological variables are summarised in Table 4. External validation of the pooled data using 
Table 1 Associations between MMP9 protein expression and clinicopathological features in the whole Cohort

\begin{tabular}{|c|c|c|c|c|c|c|}
\hline \multirow[t]{2}{*}{ Parameters } & \multicolumn{3}{|c|}{ MMP9 cytoplasmic $(\mathrm{C}+)$ expression } & \multicolumn{3}{|c|}{ MMP9 stromal (S+) expression } \\
\hline & $\begin{array}{l}\text { Negative/low } \\
\text { expression } n(\%)\end{array}$ & $\begin{array}{l}\text { High expression } \\
n(\%)\end{array}$ & $p$ value $\left(\chi^{2}\right)$ & $\begin{array}{l}\text { Negative/low } \\
\text { expression } n(\%)\end{array}$ & $\begin{array}{l}\text { High expression } \\
n(\%)\end{array}$ & $p$ value $\left(\chi^{2}\right)$ \\
\hline \multicolumn{7}{|l|}{ Age at diagnosis (years) } \\
\hline$<50$ & $172(70.5)$ & $72(29.5)$ & \multirow{2}{*}{$\begin{array}{l}0.740 \\
(0.110)\end{array}$} & $143(58.0)$ & $101(42.0)$ & \multirow{2}{*}{$\begin{array}{l}\mathbf{0 . 0 1 9} \\
(5.543)\end{array}$} \\
\hline$\geq 50$ & $309(71.7)$ & $122(28.3)$ & & $212(49.0)$ & $219(51.0)$ & \\
\hline \multicolumn{7}{|l|}{ Histological grade } \\
\hline 1 & $76(84.0)$ & $14(16.0)$ & \multirow{3}{*}{$\begin{array}{l}<\mathbf{0 . 0 0 0 1} \\
(20.570)\end{array}$} & $61(68.0)$ & $29(32.0)$ & \multirow{3}{*}{$\begin{array}{l}<\mathbf{0 . 0 0 0 1} \\
(27.165)\end{array}$} \\
\hline 2 & $177(78.0)$ & $51(22.0)$ & & $141(62.0)$ & $87(38.0)$ & \\
\hline 3 & $225(64.0)$ & $125(36.0)$ & & $153(44.0)$ & $197(56.0)$ & \\
\hline \multicolumn{7}{|l|}{ Stage } \\
\hline I & $303(74.0)$ & $105(26.0)$ & \multirow{3}{*}{$\begin{array}{l}0.081 \\
(6.743)\end{array}$} & $226(55.0)$ & $182(46.0)$ & \multirow{3}{*}{$\begin{array}{l}\mathbf{0 . 0 1 2} \\
(11.005)\end{array}$} \\
\hline II & $138(67.0)$ & $69(33.0)$ & & $106(51.0)$ & $101(19.0)$ & \\
\hline III & $37(70.0)$ & $1630.0)$ & & $23(43.0)$ & $30(57.0)$ & \\
\hline \multicolumn{7}{|l|}{ Tumour size } \\
\hline$<2.0 \mathrm{~cm}$ & $234(74.0)$ & $81(26.0)$ & \multirow{2}{*}{$\begin{array}{l}0.137 \\
(2.206)\end{array}$} & $170(54.0)$ & $145(46.0)$ & \multirow{2}{*}{$\begin{array}{l}0.604 \\
(1.269)\end{array}$} \\
\hline$\geq 2.0 \mathrm{~cm}$ & $246(69.0)$ & $110(31.0)$ & & $185(52.0)$ & $171(48.0)$ & \\
\hline \multicolumn{7}{|l|}{ Histological type } \\
\hline Ductal including NST* & $406(69.2)$ & $181(30.8)$ & \multirow{3}{*}{$\begin{array}{l}\mathbf{0 . 0 0 3} \\
(11.586)\end{array}$} & $300(51.1)$ & $287(48.9)$ & \multirow{3}{*}{$\begin{array}{l}0.092 \\
(4.88)\end{array}$} \\
\hline Lobular & $48(87.0)$ & $7(13.0)$ & & $34(62.0)$ & $21(38.0)$ & \\
\hline Special type & $22(88.0)$ & $3(12.0)$ & & $17(68.0)$ & $8(32.0)$ & \\
\hline \multicolumn{7}{|l|}{ IHC subtypes } \\
\hline ER+/HER2-low proliferation & $115(81.0)$ & $26(19.0)$ & \multirow{4}{*}{$\begin{array}{l}\mathbf{0 . 0 0 2} \\
(14.857)\end{array}$} & $85(60.3)$ & $56(40.0)$ & \multirow{4}{*}{$\begin{array}{l}\mathbf{0 . 0 0 1} \\
(15.879)\end{array}$} \\
\hline ER+/HER2- high proliferation & $154(72.0)$ & $60(28.0)$ & & $126(59.0)$ & $88(41.0)$ & \\
\hline Triple negative & $80(64.0)$ & $45(36.0)$ & & $51(40.0)$ & $74(60.0)$ & \\
\hline HER2 + & $63(62.0)$ & $39(38.2)$ & & $48(47.0)$ & $54(53.0)$ & \\
\hline \multicolumn{7}{|l|}{ Nottingham Prognostic Index } \\
\hline GPG & $155(84.0)$ & $29(16.0)$ & \multirow{3}{*}{$\begin{array}{l}<\mathbf{0 . 0 0 0 1} \\
(20.523)\end{array}$} & $117(63.0)$ & $67(37.0)$ & \multirow{3}{*}{$\begin{array}{l}\mathbf{0 . 0 0 2} \\
(12.257)\end{array}$} \\
\hline MPG & $253(68.0)$ & $122(32.0)$ & & $187(49.0)$ & $188(51.0)$ & \\
\hline PPG & $72(64.0)$ & $40(36.0)$ & & $51(46.0)$ & $61(56.0)$ & \\
\hline \multicolumn{7}{|l|}{ Lymphovascular invasion (LVI) } \\
\hline Negative/probable & $230(73.0)$ & $86(27.0)$ & \multirow{2}{*}{$\begin{array}{l}0.445 \\
(0.584)\end{array}$} & $180(57.0)$ & $136(43.0)$ & \multirow{2}{*}{$\begin{array}{l}\mathbf{0 . 0 3 1} \\
(4.669)\end{array}$} \\
\hline Definite & $157(70.0)$ & $68(30.0)$ & & $107(48.0)$ & $118(52.0)$ & \\
\hline
\end{tabular}

Significant $p$ values are highlighted in bold; GPG; Good Prognostic Group; MPG: Moderate Prognostic Group; PPG: Poor Prognostic Group

* Medullary like carcinoma was renamed as Ductal NST carcinoma according to the recent WHO book 2019 and added to the ductal NST group

BC-GenExMiner v4.0 was in agreement where high $M M P 9$ mRNA expression was associated with ER, and PR negativity, high histological grade (all; $p<0.001$ ) and poor NPI $(p<0.01)$. In PAM50 subtypes, high MMP9 expression was associated with basal-like and HER2 + classes $(p<0.0001)$.

High $M M P 9$ mRNA showed positive association with other MMPs (MMP1, MMP2, MMP7, MMP11, MMP14 and MMP15; all $p=0.0001)$, collagens [COL27A1; $(p=0.008)$, COL23A1 $(p=0.003)$ and COL11A2; $(p=0.045)], T G F \beta 1$ $(p<0.0001)$ and cytoskeletal remodelling biomarkers CDC42 ( $p=0.004$; Table 5). These associations were confirmed using BC-GenExMiner v4.0 (Supplementary Fig. 2) via gene targeted analysis.
In the METABRIC cohort, MMP9 copy number gain was associated with significantly shorter BCSS ( $p=0.016$; HR 1.3; 95\%CI 1.1-1.7; Fig. 2d). Tumours expressing high $M M P 9$ mRNA expression showed significantly shorter BCSS than the low expression subgroup $(p<0.0001$; $\mathrm{HR}=1.5$; 95\%CI 1.2-1.8; Fig. 2e). Pooled MMP9 gene expression data $(n=2071)$ in BC-GenExMiner v4.0 confirmed the association of high $M M P 9$ expression with poorer outcome ( $p=0.007$; HR $=1.3 ; 95 \% \mathrm{CI} 1.1-1.5$; Fig. 2f) and in agreement with protein expression results. In multivariate Cox regression analysis, $M M P 9$ mRNA was an independent predictor of BCSS $(p=0.048$; HR $=1.3 ; 95 \%$ CI 1.0-1.6) independent of the standard prognostic parameters of $\mathrm{BC}$ 
Table 2 Associations between MMP9 protein expression and other biomarkers in the breast cancer cohort

\begin{tabular}{|c|c|c|c|c|c|c|}
\hline \multirow[t]{2}{*}{ Parameters } & \multicolumn{3}{|c|}{ MMP9 cytoplasmic $(\mathrm{C}+)$ expression } & \multicolumn{3}{|c|}{ MMP9 stromal $(\mathrm{S}+)$ expression } \\
\hline & $\begin{array}{l}\text { Negative/low } \\
\text { expression } n(\%)\end{array}$ & High expression $n(\%)$ & $p$ value $\left(\chi^{2}\right)$ & $\begin{array}{l}\text { Negative/low } \\
\text { expression } n(\%)\end{array}$ & High expression $n(\%)$ & $p$ value $\left(\chi^{2}\right)$ \\
\hline \multicolumn{7}{|c|}{ Oestrogen (ER) status } \\
\hline Negative & $107(59.0)$ & $74(41.0)$ & \multirow{2}{*}{$\begin{array}{l}<\mathbf{0 . 0 0 0 1} \\
(18.402)\end{array}$} & $69(38.0)$ & $112(62.0)$ & \multirow{2}{*}{$\begin{array}{l}<\mathbf{0 . 0 0 0 1} \\
(20.194)\end{array}$} \\
\hline Positive & $373(76.0)$ & $118(24.0)$ & & $283(58.0)$ & $208(42.0)$ & \\
\hline \multicolumn{7}{|c|}{ Progesterone (PR) status } \\
\hline Negative & $182(66.0)$ & $95(34.0)$ & \multirow{2}{*}{$\begin{array}{l}\mathbf{0 . 0 0 5} \\
(7.860)\end{array}$} & $125(45.0)$ & $152(55.0)$ & \multirow{2}{*}{$\begin{array}{l}\mathbf{0 . 0 0 2} \\
(9.936\end{array}$} \\
\hline Positive & $284(76.0)$ & $91(24.0)$ & & $216(58.0)$ & $159(42.0)$ & \\
\hline \multicolumn{7}{|c|}{ Human epidermal growth factor receptor 2 (HER2) } \\
\hline Negative & $410(74.0)$ & $146(26.0)$ & \multirow{2}{*}{$\begin{array}{l}\mathbf{0 . 0 0 9} \\
(6.784)\end{array}$} & $302(54.0)$ & $254(46.0)$ & \multirow{2}{*}{$\begin{array}{l}0.150 \\
(2.077)\end{array}$} \\
\hline Positive & $63(61.0)$ & $40(39.0)$ & & $48(47.0)$ & $55(53.0)$ & \\
\hline \multicolumn{7}{|c|}{ Epidermal Growth Factor Receptor (EGFR) } \\
\hline Negative & $385(74.0)$ & $137(26.0)$ & \multirow{2}{*}{$\begin{array}{l}\mathbf{0 . 0 2 4} \\
(5.069)\end{array}$} & $285(55.0)$ & $237(45.0)$ & \multirow{2}{*}{$\begin{array}{l}\mathbf{0 . 0 1 6} \\
(5.856)\end{array}$} \\
\hline Positive & $85(64.0)$ & $48(36.0)$ & & $57(43.0)$ & $76(57.0)$ & \\
\hline \multicolumn{7}{|c|}{ Phosphatidylinositol-4,5-bisphosphate 3-kinase, catalytic subunit alpha (PIK3CA) } \\
\hline Negative & $93(79.0)$ & $25(21.0)$ & \multirow{2}{*}{$\begin{array}{l}\mathbf{0 . 0 5 2} \\
(3.787)\end{array}$} & $72(61.0)$ & $46(39.0)$ & \multirow{2}{*}{$\begin{array}{l}\mathbf{0 . 0 3 9} \\
(4.271)\end{array}$} \\
\hline Positive & $287(70.0)$ & $125(30.0)$ & & $207(50.0)$ & $205(50.0)$ & \\
\hline \multicolumn{7}{|l|}{ Ki67 } \\
\hline Negative & $156(77.0)$ & $47(23.0)$ & \multirow[t]{2}{*}{$\mathbf{0 . 0 5 0}(3.858))$} & $126(62.0)$ & $77(38.0)$ & \multirow{2}{*}{$\begin{array}{l}\mathbf{0 . 0 0 4} \\
(8.474)\end{array}$} \\
\hline Positive & $222(69.0)$ & $10031.0)$ & & $158(49.0)$ & $164(51.0)$ & \\
\hline \multicolumn{7}{|c|}{ Cytokeartin5/6 (CK5/6) } \\
\hline Negative & $324(71.0)$ & $127(29.0)$ & \multirow{2}{*}{$\begin{array}{l}0.080 \\
(3.071)\end{array}$} & $241(53.0)$ & $210(47.0)$ & \multirow{2}{*}{$\begin{array}{l}0.099 \\
(2.726)\end{array}$} \\
\hline Positive & $57(63.0)$ & $34(37.0)$ & & $40(44.0)$ & $51(56.0)$ & \\
\hline \multicolumn{7}{|c|}{ Cytokeartin17 (CK17) } \\
\hline Negative & $309(73.0)$ & $113(27.0)$ & \multirow{2}{*}{$\begin{array}{l}\mathbf{0 . 0 0 1} \\
(10.888)\end{array}$} & $225(53.0)$ & $197(47.0)$ & \multirow{2}{*}{$\begin{array}{l}0.395 \\
(0.724)\end{array}$} \\
\hline Positive & $42(55.0)$ & $35(45.0)$ & & $37(48.0)$ & $40(52.0)$ & \\
\hline \multicolumn{7}{|c|}{ Cell division cycle 42 (CDC42) } \\
\hline Negative & $196(76.0)$ & $60(24.0)$ & \multirow{2}{*}{$\begin{array}{l}\mathbf{0 . 0 0 3} \\
(9.046)\end{array}$} & $138(54.0)$ & $118(46.0)$ & \multirow[t]{2}{*}{$0.892(0.018)$} \\
\hline Positive & $126(64.0)$ & $72(36.0)$ & & $108(55.0)$ & $90(45.0)$ & \\
\hline Cluster of di & rentiation $44(\mathrm{CD}$ & & & & & \\
\hline Negative & $109(77.0)$ & $32(23.0)$ & 0.025 & $77(55.0)$ & $64(45.0)$ & 0.996 \\
\hline Positive & $167(67.0)$ & $84(33.0)$ & $(5.027)$ & $137(55.0)$ & $114(45.0)$ & $(0.001)$ \\
\hline
\end{tabular}

Significant $p$ values are highlighted in bold

including tumour size, histological grade, nodal stage, and proliferative fraction as assessed by Ki67.

\section{Genomic investigation and pathway analysis}

To understand the molecular biology of MMP9 protein expression as an end point, the subset of the Nottingham series that was included in the METABRIC dataset $(n=113)$ were used for DGE analysis. The dichotomisation of cases into negative/low versus high groups was based on the dichotomisation of MMP9 protein expression. Cytoplasmic MMP9 expression displayed high expression in 36/113 cases (32\%), while, stromal MMP9 showed high expression in 53/113 cases (47\%). DGE analysis identified 1630 significantly differentially expressed genes associated with cytoplasmic MMP9 expression, with decreased cytoplasmic MMP9 expression displayed 720 upregulated and 910 downregulated genes, respectively. Stromal MMP9 showed 1480 differentially regulated genes, reduced stromal MMP9 expression was associated with 667 upregulated and 813 downregulated differentially expressed genes (Fig. 3). The overlapping DGEs between (A) low Vs high MMP9 cytoplasmic expression, and (B) low Vs high MMP9 stromal expression revealed 277 upregulated differentially expressed genes and 276 downregulated differentially expressed genes (Fig. 4). The common differentially expressed genes $(n=553)$ which were significantly associated with ECM related gene ontologies (Fig. 5, Table 6). 

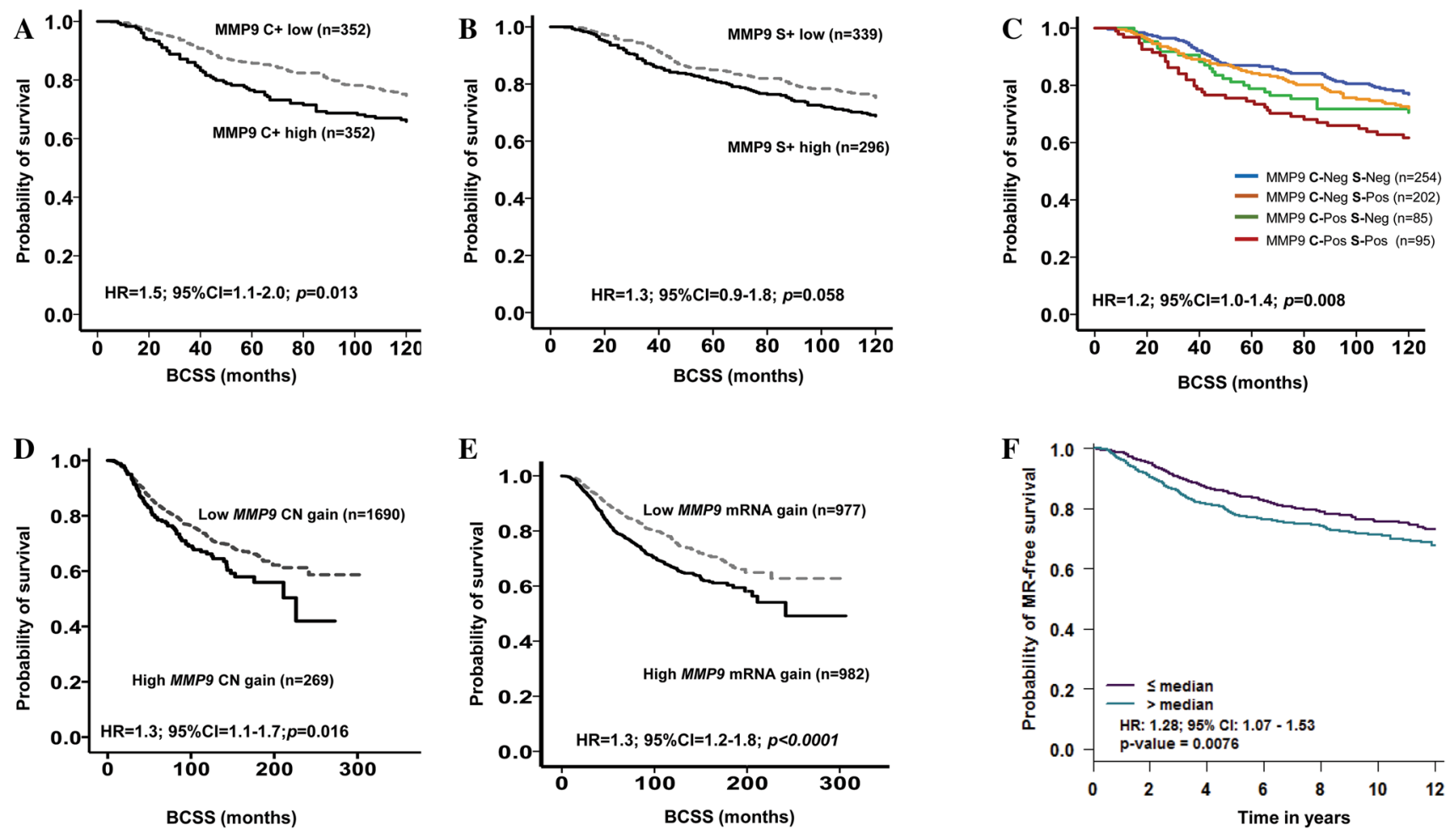

Fig. 2 Kaplan-Meier plots of MMP9 expression and breast cancer patient outcome. At protein level a cytoplasmic MMP9, b stromal MMP9, c combined cytoplasmic and stromal MMP9 expression. Combined analysis of cytoplasmic and stromal MMP9 demonstrated that tumours with high cytoplasmic and stromal expression were associated with shorter BCSS. At transcriptomic level (d) METABRIC cohort MMP9 gain, e MMP9 $m R N A$ and $\mathbf{f}$ Breast Cancer GeneExpression Miner v4.0-Kaplan-Meier plots of MMP9 gene expression. Outcome analysis revealed that high expression of $M M P 9$ was associated with shorter patient survival
Table 3 Univariate and multivariate analysis of MMP9 (C+\& S+) expression compared with tumour stage, grade, size, Ki67 and ER status for breast cancer-specific survival

\begin{tabular}{|c|c|c|c|c|c|c|}
\hline \multirow[t]{3}{*}{ Variable } & \multicolumn{6}{|c|}{ Breast cancer-specific survival } \\
\hline & \multicolumn{3}{|c|}{ Univariate } & \multicolumn{3}{|c|}{ Multivariate } \\
\hline & HR & $95 \% \mathrm{CI}$ & $p$ value & HR & $95 \% \mathrm{CI}$ & $p$ value \\
\hline \multicolumn{7}{|l|}{ Whole cohort } \\
\hline Stage & 2.7 & $1.8-3.2$ & $<0.0001$ & 2.4 & $1.8-3.2$ & $<0.0001$ \\
\hline Grade & 2.7 & $2.4-3.3$ & $<0.0001$ & 2.1 & $1.3-3.3$ & 0.001 \\
\hline Tumour size & 2.1 & $1.8-2.5$ & $<0.0001$ & 1.5 & $0.9-2.3$ & 0.068 \\
\hline ER & 1.0 & $0.9-1.1$ & $<0.0001$ & 0.9 & $0.9-1.0$ & 0.335 \\
\hline Ki67 & 2.6 & $2.1-3.3$ & $<0.0001$ & 1.5 & $0.8-2.4$ & 0.155 \\
\hline $\operatorname{MMP} 9(\mathrm{C}+)$ & 1.5 & $1.1-2.0$ & 0.013 & 1.6 & $1.1-2.3$ & 0.026 \\
\hline MMP9 (S+) & 1.3 & $1.0-1.8$ & 0.060 & 1.2 & $0.8-1.8$ & 0.305 \\
\hline
\end{tabular}

Significant $p$ values highlighted in bold

\section{Discussion}

Several studies have examined the roles of MMP9 in cancer development, progression, and its impact on patients' survival and prognosis [2, 36-38]. Limited research however has been done to demonstrate the mRNA, copy number alterations and protein expression of MMP9 in $\mathrm{BC}$ and correlate the findings with clinicopathologic variables and cytoskeletal modulators in an extended cohort of BC patients. The cytoskeleton connects the cytoplasm and the plasma membrane and responds to external stimuli and signals. Cells that grow abnormally and acquire the ability to migrate and invade are the hallmark of 


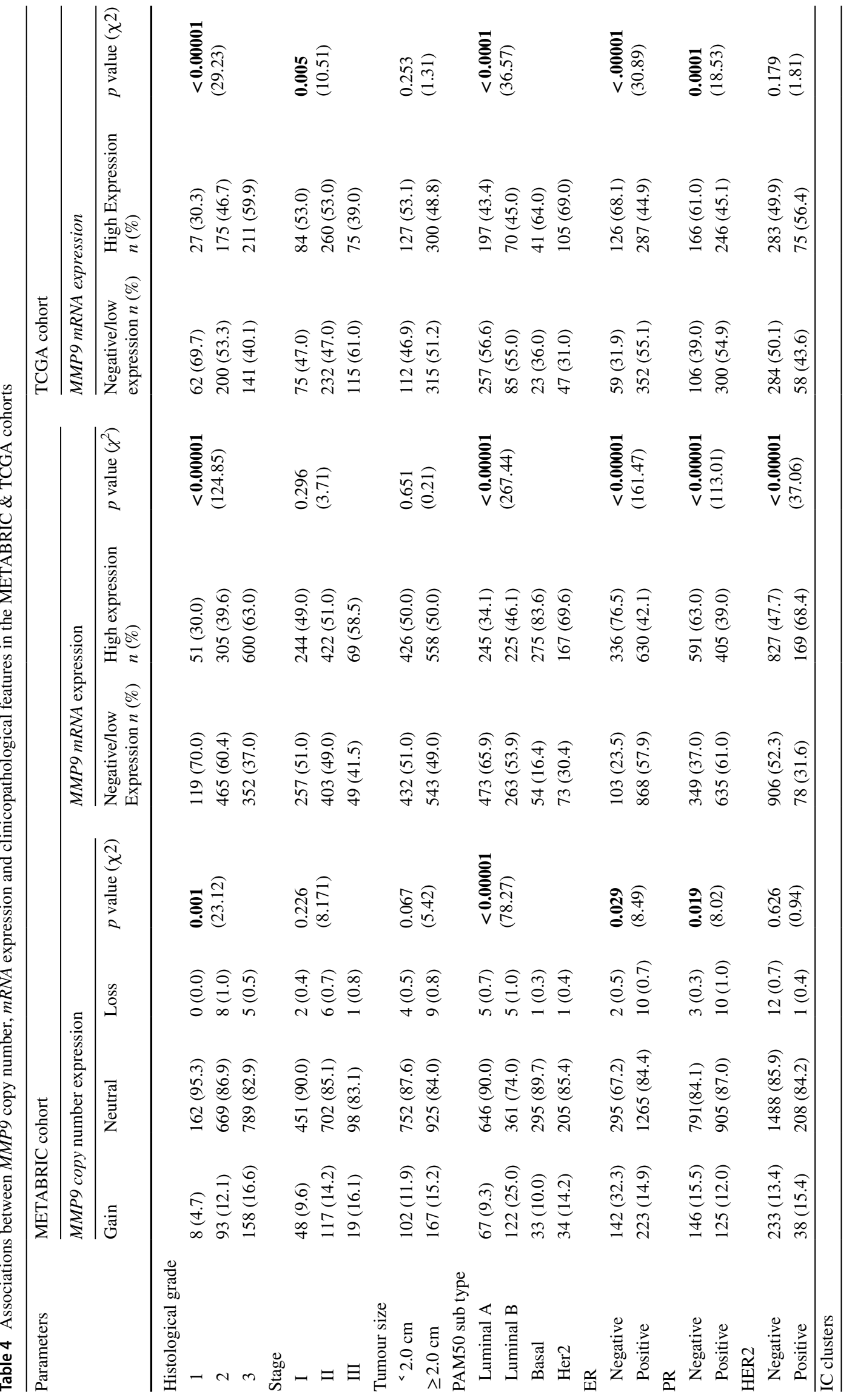




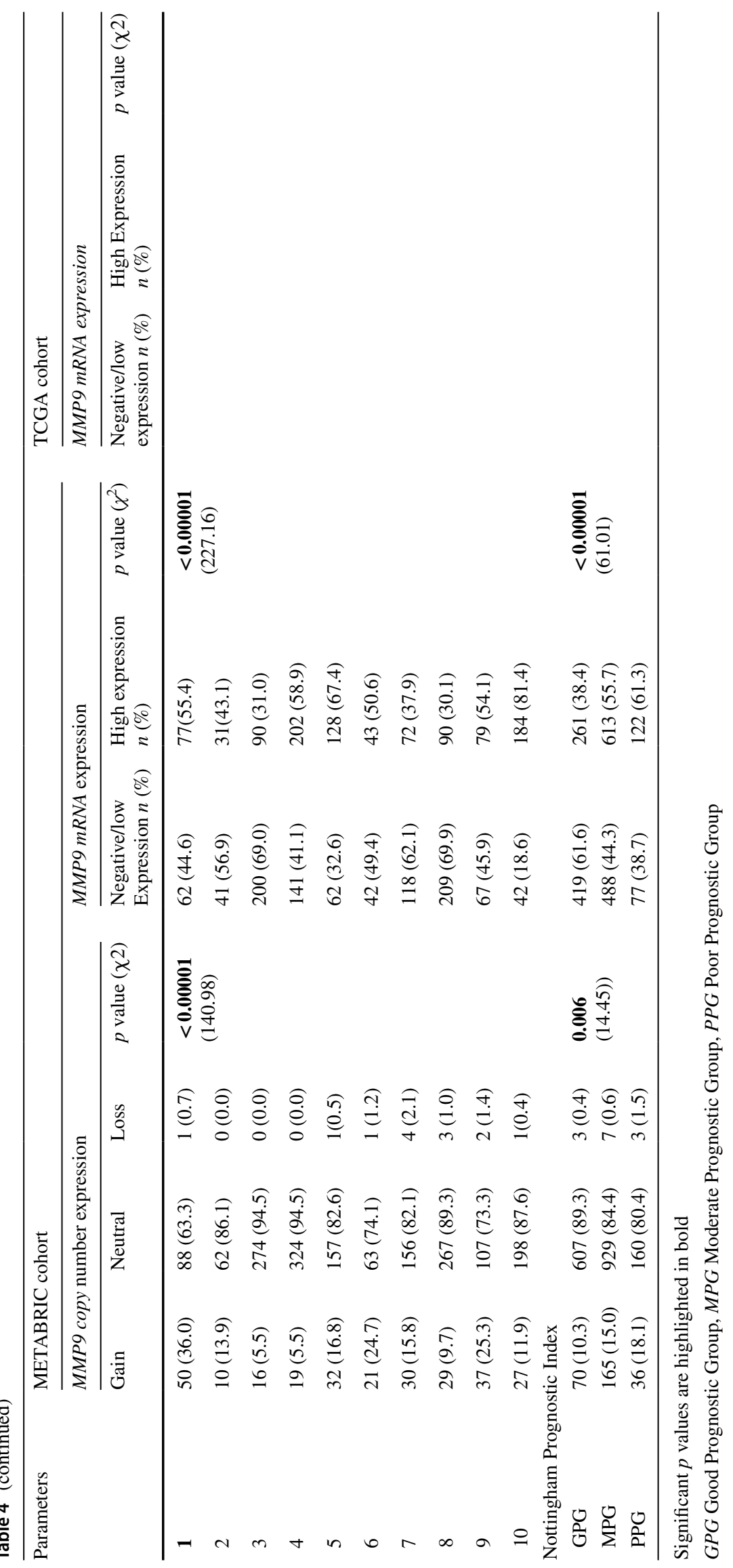


Table 5 Associations between $M M P 9 \mathrm{mRNA}$ and ECM

associated markers

\begin{tabular}{|c|c|c|c|}
\hline \multirow[t]{2}{*}{ Parameters } & \multicolumn{3}{|c|}{ METABRIC cohort } \\
\hline & $\begin{array}{l}\text { Negative/low } \\
\text { Expression } n(\%)\end{array}$ & $\begin{array}{l}\text { High Expression } \\
n(\%)\end{array}$ & $p$ value $(\chi 2)$ \\
\hline \multicolumn{4}{|c|}{ Matrix metallopeptidase 1 (MMP1) } \\
\hline Negative & $820(60.0)$ & $559(40.0)$ & \multirow[t]{2}{*}{$<\mathbf{0 . 0 0 0 1}(164.48$} \\
\hline Positive & $169(28.0)$ & $432(72.0)$ & \\
\hline \multicolumn{4}{|c|}{ Matrix metallopeptidase 2 (MMP2) } \\
\hline Negative & $604(55.0)$ & $505(45.0)$ & \multirow[t]{2}{*}{$<\mathbf{0 . 0 0 0 1}(20.548)$} \\
\hline Positive & $385(44.0)$ & $486(56.0)$ & \\
\hline \multicolumn{4}{|c|}{ Matrix metallopeptidase 7(MMP7) } \\
\hline Negative & $583(59.0)$ & $407(41.0)$ & \multirow[t]{2}{*}{$<\mathbf{0 . 0 0 0 1}(63.291)$} \\
\hline Positive & $406(41.0)$ & $584(59.0)$ & \\
\hline \multicolumn{4}{|c|}{ Matrix metallopeptidase 11 (MMP11) } \\
\hline Negative & $551(56.0)$ & $439(44.0)$ & \multirow[t]{2}{*}{$<\mathbf{0 . 0 0 0 1}(25.796)$} \\
\hline Positive & $438(44.0)$ & $552(56.0)$ & \\
\hline \multicolumn{4}{|c|}{ Matrix metallopeptidase 14 (MMP14) } \\
\hline Negative & $587(54.0)$ & $503(46.0)$ & \multirow[t]{2}{*}{$\mathbf{0 . 0 0 0 1}(14.782)$} \\
\hline Positive & $402(45.0)$ & $488(55.0)$ & \\
\hline \multicolumn{4}{|c|}{ Matrix metallopeptidase 15 (MMP15) } \\
\hline Negative & $671(55.0)$ & $544(45.0)$ & \multirow[t]{2}{*}{$<\mathbf{0 . 0 0 0 1}(35.026)$} \\
\hline Positive & $318(42.0)$ & $447(58.0)$ & \\
\hline \multicolumn{4}{|c|}{ Collagen Type XXVII Alpha 1 (COL27A1) } \\
\hline Negative & $524(53.0)$ & $466(47.0)$ & \multirow{2}{*}{$\begin{array}{l}\mathbf{0 . 0 0 8} \\
(7.032)\end{array}$} \\
\hline Positive & $465(47.0)$ & $525(53.0)$ & \\
\hline \multicolumn{4}{|c|}{ Collagen Type XXIII Alpha 1 (COL23A1) } \\
\hline Negative & $598(53.0)$ & $531(47.0)$ & \multirow[t]{2}{*}{$\mathbf{0 . 0 0 3}(8.962)$} \\
\hline Positive & $391(46.0)$ & $456(54.0)$ & \\
\hline \multicolumn{4}{|c|}{ Collagen Type XXI Alpha 2 (COL11A2) } \\
\hline Negative & $576(51.0)$ & $534(49.0)$ & \multirow[t]{2}{*}{$\mathbf{0 . 0 4 5}(4.019)$} \\
\hline Positive & $411(47.0)$ & $457(53.0)$ & \\
\hline \multicolumn{4}{|c|}{ Transforming Growth Factor Beta 1(TGFBetal) } \\
\hline Negative & $672(67.0)$ & $338(33.0)$ & \multirow{2}{*}{$\begin{array}{l}<\mathbf{0 . 0 0 0 1} \\
(226.83)\end{array}$} \\
\hline Positive & $317(32.0)$ & $653(68.0)$ & \\
\hline \multicolumn{4}{|c|}{ Cell Division Cycle 42 (CDC42) } \\
\hline Negative & $496(53.0)$ & $431(47.0)$ & \multirow[t]{2}{*}{$\mathbf{0 . 0 0 4}(8.325)$} \\
\hline Positive & $495(47.0)$ & $558(53.0)$ & \\
\hline \multicolumn{4}{|c|}{ Cell surface adhesion receptor (CD44) } \\
\hline Negative & $498(50.0)$ & $502(50.0)$ & \multirow{2}{*}{$\begin{array}{l}0.893 \\
(0.018)\end{array}$} \\
\hline Positive & $491(50.1)$ & 489 (49.9) & \\
\hline
\end{tabular}

Significant $p$ values are highlighted in bold metastatic cancers. Alteration of cytoskeletal structure is very important in cell invasion, migration, adhesion and change in morphology [39]. The degradation of ECM by MMP family members including MMP9 is believed to favour tumour growth, metastasis, invasion and cytoskeletal re-organisation [40]. Thus, the link between MMP9 and cytoskeletal modulators may have important clinical implications. The aim of this study was to determine whether elevated MMP9 expression at mRNA and protein level correlated with tumour grade, BC morphology, cytoskeletal modulators and patient outcome using a large clinical data set with long-term follow-up.

High MMP9 expression was associated with an increase in cell proliferation activity indicated by high expression of $\mathrm{Ki67}$, which is associated with poor patient outcome [41]. Also, elevated levels of CK17 was associated with increased expression of MMP9. Breast tumours expressing CK17 and CK5/6 show high mortality rate which clearly implicate a role in tailoring treatment decisions [22]. These findings strengthen the putative role of MMP9 in tumour progression. 


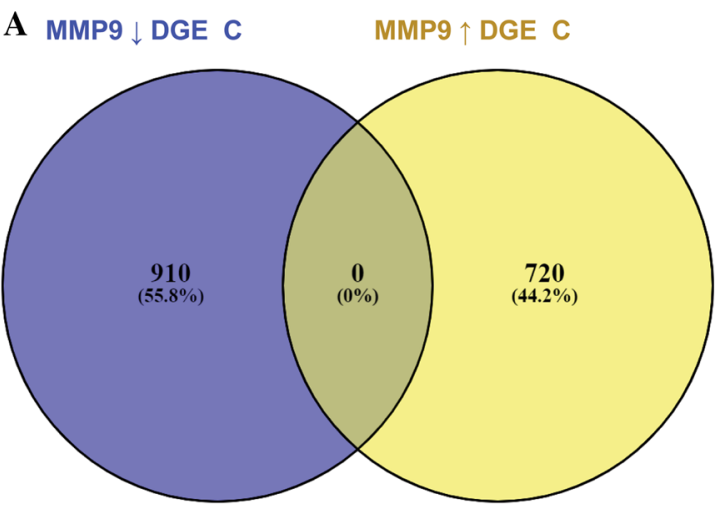

$\mathrm{N}=1630$

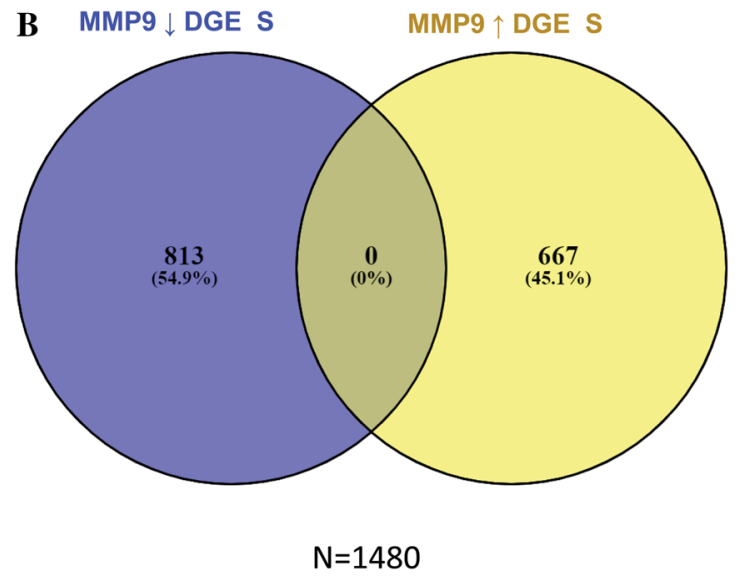

ferentially expressed genes, C: cytoplasmic expression, S: stromal expression, $(\downarrow)$ : down regulated genes, $(\uparrow)$ up regulated genes. (-) cases harbouring low expression of MMP9

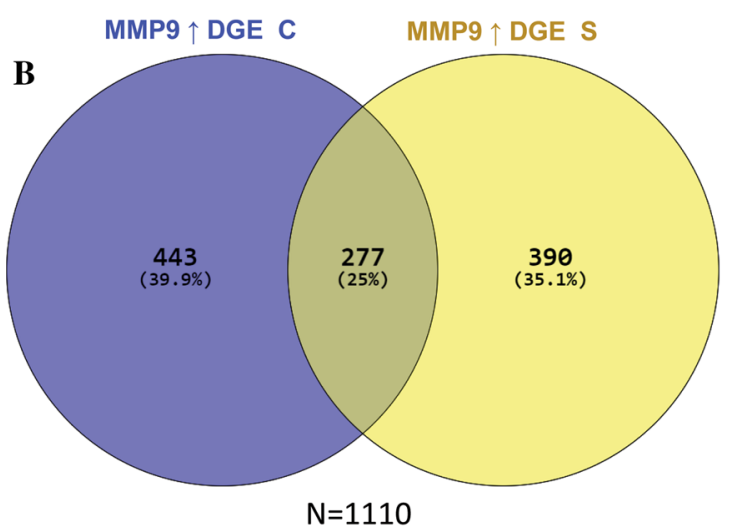

protein expression on both the cytoplasm and associated stroma of tumour cells. DGE: differentially expressed genes, C: cytoplasmic expression, S: stromal expression, $\downarrow$ : down regulated genes, $\uparrow$ up regulated genes

and vesicular trafficking [44]. A study conducted by Sipes and colleagues; revealed that, CDC42 deletion significantly reduced MMP9 activity. CDC42 is associated with formation of invadopodia, which can act as 'guiding' structures to pave the way for further cytoskeletal protrusions [45, 46]. Moreover, in vitro silencing of MMP9 decreased the migratory activity in Adenoid Cystic Carcinoma cells [47]. PIK3 and CDC42 mediate a positive feedback loop to regulate the tumour progression role [48]. Investigating the functional role of MMP9 in regulating the PIK3/CDC42 positive feedback loop in BC, might reveal a new role for MMP9 in the promotion of migration and invasion.

CD44 plays a major role in modulating migration/invasion processes during tumour advancement. Formation of CD44-MMP9 complex in prostate cancer promotes cellular such as cell migration, proliferation, cytoskeletal control 
Fig. 5 Pathway analysis for gene ontology significantly associated with cytoplasmic and stromal MMP9 protein expression. The gene panel were significantly associated with extracellular matrix related gene ontologies

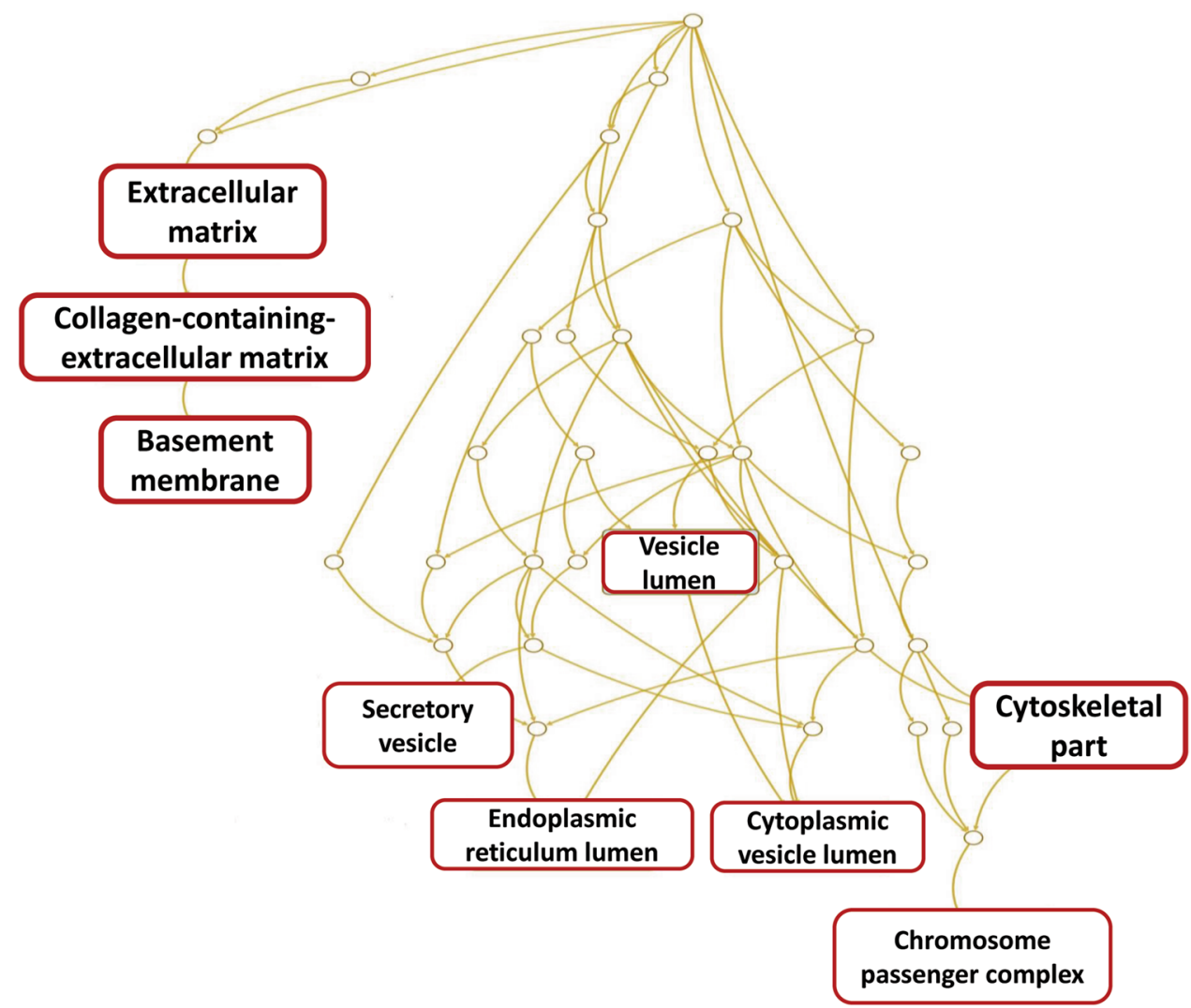

motility and ECM invasion [49]. High MMP9 expression was positively associated with CD44 in our study. MMP9 acts as a processing enzyme for CD44 which promotes cell motility and, transcriptional knockdown of MMP9 inhibits this interaction [50]. Further studies will be needed to unravel the mechanisms by which MMP9 drives CD44 mediated invasion and tumour progression.

LVI is the presence of cancer cells in lymph vessels and is linked with a poor outcome in BC [51]. Daniele et al.; [52] showed that high levels of MMP9 expression was found in $\mathrm{BC}$ tumours with positive sentinel lymph nodes. The sentinel node is the proximal lymph node affected by metastatic cells since it is the first barrier receiving lymphatic drainage from the tumour. In the event of LVI, interactions between ECM and stromal non-tumoural cells induce migration, invasion and metastasis. Moreover, stromal fibroblast reported to secrete MMP9, which in turn may activate tumour cells [53]. Lymphatic networks within lymph nodes spread out before the onset of metastasis [54]. Although in our study we observed a weak association with stromal MMP9 and LVI, it warrants further investigations. Evaluation of stromal MMP9 expression may provide valuable information regarding the early LVI events.

The oncogenic expression of HER2 induces BC disease progression and invasiveness, which is hypothesised to increase MMP9 activity [53]. In this study, high MMP9
mRNA expression was associated with poor prognostic parameters including higher tumour grade, ER-/PR-, HER $2+$ tumours and TNBC tumours. MMP9 was also highly expressed in basal type tumours over luminal A and luminal B tumours. This is consistent with another study which found MMP9 as differentially expressed between molecular subsets of tumours and as a feature of TNBC and HER $2+\mathrm{BCs}$ [55]. Increased MMP9 expression was associated with the poor prognostic category of the NPI. Hence, as observed in our study, MMP9 is a marker indicative of unfavourable prognosis in BC. Stromal invasion requires degradation of the basement membrane. MMP9 cleave the basement membrane type IV collagen and promote tumour invasion and metastasis [56, 57]. The correlation between high expression of MMP9 and collagen type XXVII $\alpha 1, X X I I I \alpha 1, X X I \alpha 2$ may also induce basement degradation. Collagens, matricellular proteins and CDC42 at mRNA level showed strong positive association with $M M P 9$. Results on pathway analysis confirmed the significant association with collagens, extracellular matrix and cytoskeletal part gene ontologies. This implies that MMP9 plays a role in tumourigenic pathways and could be a marker of poor prognosis in $\mathrm{BC}$.

This study reveals that MMP9 at both proteomic and transcriptomic levels is associated with poor prognostic characteristics and short-term survival outcomes in BC. Cytoplasmic MMP9 expression on its own and combined 
Table 6 Pathway analysis for gene ontology significantly associated with cytoplasmic and stromal MMP9 protein expression

\begin{tabular}{|c|c|c|c|c|c|c|c|c|c|}
\hline Ontology & Name & $\begin{array}{l}\text { Genes in } \\
\text { Ontol- } \\
\text { ogy }\end{array}$ & Observed & Expected & & Enrichment & $p$ value & $\begin{array}{l}\text { False discovery } \\
\text { rate }\end{array}$ & Genes \\
\hline GO:0,031,012 & Extracellular Matrix & 496 & 37 & 10.065 & 3.6761 & & $<0.0001$ & $<0.0001$ & $\begin{array}{l}\text { CAN, AMTN, } \\
\text { AZGP1, CDH2, } \\
\text { CHAD, CILP, } \\
\text { COCH, COL16A1, } \\
\text { COL17A1, } \\
\text { COL4A5 }\end{array}$ \\
\hline GO:0,062,023 & $\begin{array}{l}\text { Collagen-Contain- } \\
\text { ing Extracellular } \\
\text { Matrix }\end{array}$ & 366 & 28 & 7.4269 & 3.7701 & & $<0.0001$ & $<0.0001$ & $\begin{array}{l}\text { CAN, AMTN, } \\
\text { AZGP1, CDH2, } \\
\text { CHAD, CILP, } \\
\text { COCH, COL16A1, } \\
\text { COL17A1, } \\
\text { COL4A5 }\end{array}$ \\
\hline GO:0,005,604 & $\begin{array}{l}\text { Basement Mem- } \\
\text { brane }\end{array}$ & 91 & 10 & 1.8466 & 5.454 & & $<0.0001$ & 0.003 & $\begin{array}{l}\text { ACAN, AMTN, } \\
\text { COL17A1, } \\
\text { COLAA5, COL9A2, } \\
\text { FBLN1, LAD1, } \\
\text { LAMA3, NTN4, } \\
\text { THBS4 }\end{array}$ \\
\hline GO:0,005,788 & $\begin{array}{l}\text { Endoplasmic } \\
\text { Reticulum Lumen }\end{array}$ & 306 & 19 & 6.8182 & 3.0599 & & $<0.0001$ & 0.003 & $\begin{array}{l}\text { AMTN, BCHE, } \\
\text { CDH2, CESA, } \\
\text { CHGB, COL16A1, } \\
\text { COL17A1, } \\
\text { COL4A5, COL9A2, } \\
\text { ERAP2 }\end{array}$ \\
\hline GO:0,044,430 & Cytoskeletal Part & 1620 & 57 & 32.873 & 1.7339 & & $<0.0001$ & 0.004 & $\begin{array}{l}\text { ACTA1, ACTG2, } \\
\text { ACTR3, AK5, } \\
\text { AURKA, AURKB, } \\
\text { BIRC5, CASP14, } \\
\text { CCNB1, CCNE1 }\end{array}$ \\
\hline GO:0,031,983 & Vesicle Lumen & 337 & 19 & 6.8385 & 2.7784 & & $<0.0001$ & 0.008 & $\begin{array}{l}C F D, F A B P 5, G G H, \\
\text { GLA, GNLY, } \\
\text { LCN2, LYZ, } \\
\text { PLFM4, ORM1, } \\
\text { PNP }\end{array}$ \\
\hline GO:0,032,133 & $\begin{array}{c}\text { Chromosome Pas- } \\
\text { senger Complex }\end{array}$ & 5 & 3 & 0.10146 & 29.568 & & $<0.0001$ & 0.009 & $\begin{array}{l}A U R K A, A U R K B, \\
\quad \text { BIRC5 }\end{array}$ \\
\hline
\end{tabular}

Significant $p$ values are highlighted in bold

cytoplasmic and stromal expression was predicative for shorter BCSS in the whole cohort, MMP9 did not show any association with patient outcome. Stromal cell genetic stability plays significant role in modulating the tumour microenvironment contributing to drug resistance and tumour relapse [58]. Moreover, expression of tumour markers in the stroma is found to be closely associated with tumour progression and patient outcome [59]. These findings suggest that MMP9 in both stromal cells and tumour cells might play an important role in the BC progression. In agreement with our study high expression of MMP9 was associated with poor patient survival [60]. Thus, the current study provides definitive evidence that MMP9 is an independent prognostic marker of poor short-term clinical outcome in primary BC and supports further mechanistic and translational studies to target MMP9.

Acknowledgements We thank the Nottingham Health Science Biobank and Breast Cancer Now Tissue Bank for the provision of tissue samples.

Funding This research received no specific grant from any funding agency in the public, commercial, or not-for-profit sectors.

\section{Compliance with ethical standards}

Conflict of interest All authors declare that they have no conflicts of interest. 
Research involving human participants This article does not contain any studies with human participants or animals performed by any of the authors. All procedures performed in studies involving human participants were in accordance with the ethical standards of the institutional and/or national research committee and with the 1964 Helsinki declaration and its later amendments or comparable ethical standards.

Informed consent This work obtained ethics approval to use the human tissue samples by the North West - Greater Manchester Central Research Ethics Committee under the title Nottingham Health Science Biobank (NHSB), reference number 15/NW/0685. Informed consent was obtained from all individuals prior to surgery to use their tissue materials in research. This study was performed according to the REMARK guidelines for tumour prognostic studies.

Availability of data and materials The authors confirm the data that has been used in this work are available on reasonable request.

Open Access This article is licensed under a Creative Commons Attribution 4.0 International License, which permits use, sharing, adaptation, distribution and reproduction in any medium or format, as long as you give appropriate credit to the original author(s) and the source, provide a link to the Creative Commons licence, and indicate if changes were made. The images or other third party material in this article are included in the article's Creative Commons licence, unless indicated otherwise in a credit line to the material. If material is not included in the article's Creative Commons licence and your intended use is not permitted by statutory regulation or exceeds the permitted use, you will need to obtain permission directly from the copyright holder. To view a copy of this licence, visit http://creativecommons.org/licenses/by/4.0/.

\section{References}

1. Gialeli C, Theocharis AD, Karamanos NK (2011) Roles of matrix metalloproteinases in cancer progression and their pharmacological targeting. FEBS J 278(1):16-27. https://doi.org/10.111 1/j.1742-4658.2010.07919.x

2. Mehner C, Hockla A, Miller E, Ran S, Radisky DC, Radisky ES (2014) Tumor cell-produced matrix metalloproteinase 9 (MMP-9) drives malignant progression and metastasis of basal-like triple negative breast cancer. Oncotarget 5(9):2736-2749. https://doi. org/10.18632/oncotarget.1932

3. Nagase H, Visse R, Murphy G (2006) Structure and function of matrix metalloproteinases and TIMPs. Cardiovasc Res 69(3):562573. https://doi.org/10.1016/j.cardiores.2005.12.002

4. Christensen J, Shastri VP (2015) Matrix-metalloproteinase-9 is cleaved and activated by cathepsin K. BMC Res Notes 8:322. https://doi.org/10.1186/s13104-015-1284-8

5. Chantrain CF, Shimada H, Jodele S, Groshen S, Ye W, Shalinsky DR, Werb Z, Coussens LM, DeClerck YA (2004) Stromal matrix metalloproteinase-9 regulates the vascular architecture in neuroblastoma by promoting pericyte recruitment. Can Res 64(5):1675-1686

6. Gorden DL, Fingleton B, Crawford HC, Jansen DE, Lepage M, Matrisian LM (2007) Resident stromal cell-derived MMP-9 promotes the growth of colorectal metastases in the liver microenvironment. Int J Cancer 121(3):495-500. https://doi.org/10.1002/ ijc. 22594

7. Shi H, Wu Y, Wang Y, Zhou M, Yan S, Chen Z, Gu D, Cai Y (2015) Liquiritigenin potentiates the inhibitory effects of cisplatin on invasion and metastasis via downregulation MMP-2/9 and PI3 $\mathrm{K} / \mathrm{AKT}$ signaling pathway in $\mathrm{B} 16 \mathrm{~F} 10$ melanoma cells and mice model. Nutr Cancer 67(5):761-770. https://doi.org/10.1080/01635 581.2015.1037962

8. Majumder A, Ray S, Banerji A (2019) Epidermal growth factor receptor-mediated regulation of matrix metalloproteinase- 2 and matrix metalloproteinase- 9 in MCF-7 breast cancer cells. Mol Cell Biochem 452(1-2):111-121. https://doi.org/10.1007/s1101 0-018-3417-6

9. Cohen M, Wuillemin C, Irion O, Bischof P (2008) Regulation of MMP-9 by p53 in first trimester cytotrophoblastic cells. Hum Reprod 23(10):2273-2281. https://doi.org/10.1093/humrep/den26 4

10. Bouzahzah B, Albanese C, Ahmed F, Pixley F, Lisanti MP, Segall JD, Condeelis J, Joyce D, Minden A, Der CJ, Chan A, Symons M, Pestell RG (2001) Rho family GTPases regulate mammary epithelium cell growth and metastasis through distinguishable pathways. Mol Med 7(12):816-830

11. Poincloux R, Lizarraga F, Chavrier P (2009) Matrix invasion by tumour cells: a focus on MT1-MMP trafficking to invadopodia. J Cell Sci 122(Pt 17):3015-3024. https://doi.org/10.1242/ jes. 034561

12. Gupta A, Cao W, Sadashivaiah K, Chen W, Schneider A, Chellaiah MA (2013) Promising noninvasive cellular phenotype in prostate cancer cells knockdown of matrix metalloproteinase 9. Sci World J 2013:493689. https://doi.org/10.1155/2013/493689

13. van 't Veer LJ, Dai H, van de Vijver MJ, He YD, Hart AA, Mao M, Peterse HL, van der Kooy K, Marton MJ, Witteveen AT, Schreiber GJ, Kerkhoven RM, Roberts C, Linsley PS, Bernards R, Friend SH (2002) Gene expression profiling predicts clinical outcome of breast cancer. Nature 415 (6871):530-536. https:// doi.org/10.1038/415530a

14. McGowan PM, Duffy MJ (2008) Matrix metalloproteinase expression and outcome in patients with breast cancer: analysis of a published database. Ann Oncol 19(9):1566-1572. https:// doi.org/10.1093/annonc/mdn180

15. Joseph C, Macnamara O, Craze M, Russell R, Provenzano E, Nolan CC, Diez-Rodriguez M, Sonbul SN, Aleskandarany MA, Green AR, Rakha EA, Ellis IO, Mukherjee A (2018) Mediator complex (MED) 7: a biomarker associated with good prognosis in invasive breast cancer, especially ER+ luminal subtypes. Br J Cancer 118(8):1142-1151. https://doi.org/10.1038/s4141 6-018-0041-x

16. WHO (2019) The classification of tumours editorial board: breast tumours, 5th edn. World Health Organization, IARC Press, Lyon

17. Muftah AA, Aleskandarany MA, Al-Kaabi MM, Sonbul SN, Diez-Rodriguez M, Nolan CC, Caldas C, Ellis IO, Rakha EA, Green AR (2017) Ki67 expression in invasive breast cancer: the use of tissue microarrays compared with whole tissue sections. Breast Cancer Res Treat 164(2):341-348. https://doi.org/10.1007/ s10549-017-4270-0

18. Abd El-Rehim DM, Pinder SE, Paish CE, Bell JA, Rampaul RS, Blamey RW, Robertson JF, Nicholson RI, Ellis IO (2004) Expression and co-expression of the members of the epidermal growth factor receptor (EGFR) family in invasive breast carcinoma. Br J Cancer 91(8):1532-1542. https://doi.org/10.1038/sj.bjc.6602184

19. Chrysanthou E, Gorringe KL, Joseph C, Craze M, Nolan CC, Diez-Rodriguez M, Green AR, Rakha EA, Ellis IO, Mukherjee A (2017) Phenotypic characterisation of breast cancer: the role of CDC42. Breast Cancer Res Treat 164(2):317-325. https://doi. org/10.1007/s10549-017-4267-8

20. Ahmed MA, Aleskandarany MA, Rakha EA, Moustafa RZ, Benhasouna A, Nolan C, Green AR, Ilyas M, Ellis IO (2012) A $\mathrm{CD} 44(-) / \mathrm{CD} 24(+)$ phenotype is a poor prognostic marker in early invasive breast cancer. Breast Cancer Res Treat 133(3):979-995. https://doi.org/10.1007/s10549-011-1865-8 
21. Aleskandarany MA, Rakha EA, Ahmed MA, Powe DG, Paish EC, Macmillan RD, Ellis IO, Green AR (2010) PIK3CA expression in invasive breast cancer: a biomarker of poor prognosis. Breast Cancer Res Treat 122(1):45-53. https://doi.org/10.1007/s1054 9-009-0508-9

22. Abd El-Rehim DM, Pinder SE, Paish CE, Bell J, Blamey RW, Robertson JF, Nicholson RI, Ellis IO (2004) Expression of luminal and basal cytokeratins in human breast carcinoma. J Pathol 203(2):661-671. https://doi.org/10.1002/path.1559

23. Goldhirsch A, Winer EP, Coates AS, Gelber RD, Piccart-Gebhart M, Thurlimann B, Senn HJ, Panel m (2013) Personalizing the treatment of women with early breast cancer: highlights of the St Gallen International Expert Consensus on the Primary Therapy of Early Breast Cancer 2013. Ann Oncol 24(9):2206-2223. https ://doi.org/10.1093/annonc/mdt303

24. Curtis C, Shah SP, Chin SF, Turashvili G, Rueda OM, Dunning MJ, Speed D, Lynch AG, Samarajiwa S, Yuan Y, Graf S, Ha G, Haffari G, Bashashati A, Russell R, McKinney S, Group M, Langerod A, Green A, Provenzano E, Wishart G, Pinder S, Watson P, Markowetz F, Murphy L, Ellis I, Purushotham A, BorresenDale AL, Brenton JD, Tavare S, Caldas C, Aparicio S (2012) The genomic and transcriptomic architecture of 2,000 breast tumours reveals novel subgroups. Nature 486(7403):346-352. https://doi. org/10.1038/nature10983

25. Jezequel P, Campone M, Gouraud W, Guerin-Charbonnel C, Leux C, Ricolleau G, Campion L (2012) bc-GenExMiner: an easy-touse online platform for gene prognostic analyses in breast cancer. Breast Cancer Res Treat 131(3):765-775. https://doi.org/10.1007/ s10549-011-1457-7

26. Cancer Genome Atlas N (2012) Comprehensive molecular portraits of human breast tumours. Nature 490(7418):61-70. https:// doi.org/10.1038/nature11412

27. Joseph C, Arshad M, Kurozomi S, Althobiti M, Miligy IM, Al-Izzi S, Toss MS, Goh FQ, Johnston SJ, Martin SG, Ellis IO, Mongan NP, Green AR, Rakha EA (2019) Overexpression of the cancer stem cell marker CD133 confers a poor prognosis in invasive breast cancer. Breast Cancer Res Treat 174(2):387-399. https:// doi.org/10.1007/s10549-018-05085-9

28. Abd El-Rehim DM, Ball G, Pinder SE, Rakha E, Paish C, Robertson JF, Macmillan D, Blamey RW, Ellis IO (2005) High-throughput protein expression analysis using tissue microarray technology of a large well-characterised series identifies biologically distinct classes of breast cancer confirming recent cDNA expression analyses. Int J Cancer 116(3):340-350. https://doi.org/10.1002/ ijc. 21004

29. McShane LM, Altman DG, Sauerbrei W, Taube SE, Gion M, Clark GM, Statistics Subcommittee of the NCIEWGoCD (2005) REporting recommendations for tumour MARKer prognostic studies (REMARK). Br J Cancer 93(4):387-391. https://doi. org/10.1038/sj.bjc.6602678

30. Lohse M, Bolger AM, Nagel A, Fernie AR, Lunn JE, Stitt M, Usadel B (2012) RobiNA: a user-friendly, integrated software solution for RNA-Seq-based transcriptomics. Nucleic Acids Res 40(Web Server issue):W622-627. https://doi.org/10.1093/nar/ gks540

31. Wang J, Vasaikar S, Shi Z, Greer M, Zhang B (2017) WebGestalt 2017: a more comprehensive, powerful, flexible and interactive gene set enrichment analysis toolkit. Nucleic Acids Res 45(W1):W130-W137. https://doi.org/10.1093/nar/gkx356

32. Zhang B, Kirov S, Snoddy J (2005) WebGestalt: an integrated system for exploring gene sets in various biological contexts. Nucleic Acids Res 33(Web Server issue):W741-W748. https:// doi.org/10.1093/nar/gki475

33. Pirooznia M, Nagarajan V, Deng Y (2007) GeneVenn-a web application for comparing gene lists using Venn diagrams.
Bioinformation 1(10):420-422. https://doi.org/10.6026/97320 630001420

34. Cerami E, Gao J, Dogrusoz U, Gross BE, Sumer SO, Aksoy BA, Jacobsen A, Byrne CJ, Heuer ML, Larsson E, Antipin Y, Reva B, Goldberg AP, Sander C, Schultz N (2012) The cBio cancer genomics portal: an open platform for exploring multidimensional cancer genomics data. Cancer Discov 2(5):401-404. https://doi. org/10.1158/2159-8290.CD-12-0095

35. Gao J, Aksoy BA, Dogrusoz U, Dresdner G, Gross B, Sumer SO, Sun Y, Jacobsen A, Sinha R, Larsson E, Cerami E, Sander C, Schultz N (2013) Integrative analysis of complex cancer genomics and clinical profiles using the cBioPortal. Science Signal 6(269):11. https://doi.org/10.1126/scisignal.2004088

36. Pego ER, Fernandez I, Nunez MJ (2018) Molecular basis of the effect of MMP-9 on the prostate bone metastasis: a review. Urol Oncol 36(6):272-282. https://doi.org/10.1016/j.urolo nc.2018.03.009

37. Shao W, Wang W, Xiong XG, Cao C, Yan TD, Chen G, Chen H, Yin W, Liu J, Gu Y, Mo M, He J (2011) Prognostic impact of MMP-2 and MMP-9 expression in pathologic stage IA nonsmall cell lung cancer. J Surg Oncol 104(7):841-846. https://doi. org $/ 10.1002 /$ jso. 22001

38. Hu X, Li D, Zhang W, Zhou J, Tang B, Li L (2012) Matrix metalloproteinase-9 expression correlates with prognosis and involved in ovarian cancer cell invasion. Arch Gynecol Obstet 286(6):1537-1543. https://doi.org/10.1007/s00404-012-2456-6

39. Birukova AA, Smurova K, Birukov KG, Usatyuk P, Liu F, Kaibuchi K, Ricks-Cord A, Natarajan V, Alieva I, Garcia JG, Verin AD (2004) Microtubule disassembly induces cytoskeletal remodeling and lung vascular barrier dysfunction: role of Rho-dependent mechanisms. J Cell Physiol 201(1):55-70. https://doi.org/10.1002/ jcp. 20055

40. Jablonska-Trypuc A, Matejczyk M, Rosochacki S (2016) Matrix metalloproteinases (MMPs), the main extracellular matrix (ECM) enzymes in collagen degradation, as a target for anticancer drugs. J Enzyme Inhib Med Chem 31(sup1):177-183. https://doi. org/10.3109/14756366.2016.1161620

41. Yerushalmi R, Woods R, Ravdin PM, Hayes MM, Gelmon KA (2010) Ki67 in breast cancer: prognostic and predictive potential. Lancet Oncol 11(2):174-183. https://doi.org/10.1016/S1470 -2045(09)70262-1

42. Davis NM, Sokolosky M, Stadelman K, Abrams SL, Libra M, Candido S, Nicoletti F, Polesel J, Maestro R, D’Assoro A, Drobot L, Rakus D, Gizak A, Laidler P, Dulinska-Litewka J, Basecke J, Mijatovic S, Maksimovic-Ivanic D, Montalto G, Cervello M, Fitzgerald TL, Demidenko Z, Martelli AM, Cocco L, Steelman LS, McCubrey JA (2014) Deregulation of the EGFR/PI3K/ PTEN/Akt/mTORC1 pathway in breast cancer: possibilities for therapeutic intervention. Oncotarget 5(13):4603-4650. https://doi. org/10.18632/oncotarget.2209

43. Chen JS, Wang Q, Fu XH, Huang XH, Chen XL, Cao LQ, Chen LZ, Tan HX, Li W, Bi J, Zhang LJ (2009) Involvement of PI3K/ PTEN/AKT/mTOR pathway in invasion and metastasis in hepatocellular carcinoma: Association with MMP-9. Hepatol Res 39(2):177-186. https://doi.org/10.1111/j.1872-034X.2008.00449 .x

44. Zhang Y, Li J, Lai XN, Jiao XQ, Xiong JP, Xiong LX (2019) Focus on $\mathrm{Cdc} 42$ in breast cancer: new insights, target therapy development and non-coding RNAs. Cells. https://doi.org/10.3390/cells 8020146

45. Linder S (2007) The matrix corroded: podosomes and invadopodia in extracellular matrix degradation. Trends Cell Biol 17(3):107-117. https://doi.org/10.1016/j.tcb.2007.01.002 
46. Weaver AM (2006) Invadopodia: specialized cell structures for cancer invasion. Clin Exp Metas 23(2):97-105. https://doi. org/10.1007/s10585-006-9014-1

47. Nascimento CF, Gama-De-Souza LN, Freitas VM, Jaeger RG (2010) Role of MMP9 on invadopodia formation in cells from adenoid cystic carcinoma. Study by laser scanning confocal microscopy. Microsc Res Tech 73(2):99-108. https://doi. org/10.1002/jemt.20761

48. Nakahara H, Otani T, Sasaki T, Miura Y, Takai Y, Kogo M (2003) Involvement of $\mathrm{Cdc} 42$ and $\mathrm{Rac}$ small $\mathrm{G}$ proteins in invadopodia formation of RPMI7951 cells. Genes Cells 8(12):1019-1027

49. Desai B, Ma T, Chellaiah MA (2008) Invadopodia and matrix degradation, a new property of prostate cancer cells during migration and invasion. J Biol Chem 283(20):13856-13866. https://doi. org/10.1074/jbc.M709401200

50. Chetty C, Vanamala SK, Gondi CS, Dinh DH, Gujrati M, Rao JS (2012) MMP-9 induces CD44 cleavage and CD44 mediated cell migration in glioblastoma xenograft cells. Cell Signal 24(2):549_ 559. https://doi.org/10.1016/j.cellsig.2011.10.008

51. Lee AK, DeLellis RA, Silverman ML, Heatley GJ, Wolfe HJ (1990) Prognostic significance of peritumoral lymphatic and blood vessel invasion in node-negative carcinoma of the breast. J Clin Oncol 8(9):1457-1465. https://doi.org/10.1200/ JCO.1990.8.9.1457

52. Daniele A, Zito AF, Giannelli G, Divella R, Asselti M, Mazzocca A, Paradiso A, Quaranta M (2010) Expression of metalloproteinases MMP-2 and MMP-9 in sentinel lymph node and serum of patients with metastatic and non-metastatic breast cancer. Anticancer Res 30(9):3521-3527

53. Pellikainen JM, Ropponen KM, Kataja VV, Kellokoski JK, Eskelinen MJ, Kosma VM (2004) Expression of matrix metalloproteinase (MMP)-2 and MMP-9 in breast cancer with a special reference to activator protein-2, HER2, and prognosis. Clin Cancer Res 10(22):7621-7628. https://doi.org/10.1158/1078-0432. CCR-04-1061

54. Achen MG, Stacker SA (2008) Molecular control of lymphatic metastasis. Ann N Y Acad Sci 1131:225-234. https://doi. org/10.1196/annals.1413.020
55. Remacle AG, Noel A, Duggan C, McDermott E, O'Higgins N, Foidart JM, Duffy MJ (1998) Assay of matrix metalloproteinases types 1, 2, 3 and 9 in breast cancer. Br J Cancer 77(6):926-931. https://doi.org/10.1038/bjc.1998.153

56. Radisky ES, Radisky DC (2010) Matrix metalloproteinaseinduced epithelial-mesenchymal transition in breast cancer. J Mamm Gland Biol Neoplasia 15(2):201-212. https://doi. org/10.1007/s10911-010-9177-x

57. Iochmann S, Blechet C, Chabot V, Saulnier A, Amini A, Gaud G, Gruel Y, Reverdiau P (2009) Transient RNA silencing of tissue factor pathway inhibitor-2 modulates lung cancer cell invasion. Clin Exp Metas 26(5):457-467. https://doi.org/10.1007/s1058 5-009-9245-Z

58. Quail DF, Joyce JA (2013) Microenvironmental regulation of tumor progression and metastasis. Nat Med 19(11):1423-1437. https://doi.org/10.1038/nm.3394

59. Labovsky V, Martinez LM, Davies KM, Garcia-Rivello H, Calcagno Mde L, Matas A, Fernandez Vallone VB, Wernicke A, Choi H, Chasseing NA (2015) Association between ligands and receptors related to the progression of early breast cancer in tumor epithelial and stromal cells. Clin Breast Cancer 15(1):e13-21. https://doi.org/10.1016/j.clbc.2014.05.006

60. Li HC, Cao DC, Liu Y, Hou YF, Wu J, Lu JS, Di GH, Liu G, Li FM, Ou ZL, Jie C, Shen ZZ, Shao ZM (2004) Prognostic value of matrix metalloproteinases (MMP-2 and MMP-9) in patients with lymph node-negative breast carcinoma. Breast Cancer Res Treat 88(1):75-85. https://doi.org/10.1007/s10549-004-1200-8

Publisher's Note Springer Nature remains neutral with regard to jurisdictional claims in published maps and institutional affiliations. 